\title{
WILEY-VCH
}

This is the peer reviewed version of the following article: E. D. Finlayson, I. R. Hooper, C. R. Lawrence, M. Heath, D. Anderson, J. R. Sambles, P. Vukusic, Advanced Optical Materials 2018, 1700843. https://doi.org/10.1002/adom.201700843, which has been published in final form at 10.1002/adom.201700843. This article may be used for non-commercial purposes in accordance with Wiley Terms and Conditions for Self-Archiving.

Article type: Communication

\section{Covert Images using Surface Plasmon-Mediated Optical Polarization Conversion}

Ewan D. Finlayson*, Ian R. Hooper, Christopher R. Lawrence, Mark Heath, David Anderson, J. Roy Sambles and Peter Vukusic

Dr E. D. Finlayson, Dr I. R. Hooper, M. Heath, D. Anderson, Prof. J. R. Sambles, Prof. P. Vukusic

Department of Physics and Astronomy, University of Exeter, EX4 4QL, United Kingdom E.Finlayson@exeter.ac.uk

Prof. C. R. Lawrence

QinetiQ, Cody Technology Park, Farnborough, GU14 0LX, United Kingdom

Keywords: plasmonic colour pixels; nanophotonics; displays; optical polarization; anticounterfeiting

Covert optical signatures are a vital element in anti-counterfeiting technologies. ${ }^{[1]}$ Plasmonic surfaces offer a means of manipulating the properties of light ${ }^{[2-5]}$ including the realization of coloured pixels and images. ${ }^{[6-19]}$ In this work we demonstrate concealed images with accurate colour reproduction using plasmonic pixel arrays. The spectral and spatial control of optical polarization conversion is accomplished by tailoring the interaction of light with surface plasmons through the design and arrangement of surface nanostructures. The latent image is revealed using a polarization-sensitive optical system, which represents a means for the authentication of security features that can be created using these nanostructured devices. A red-green-blue colour space is defined containing a wide gamut of chromaticities, enabling comprehensive full-colour image capability. The device concept extends the functionality of a polarization-dependent plasmonic response to realise the encoding of a colour image in covert form.

Surface plasmon polaritons comprise electromagnetic fields coupled to charge oscillations at the interface between a conductor and a dielectric. ${ }^{[2,3]}$ At optical frequencies, nanostructuring can be used to facilitate coupling between surface plasmons and free-space 


\section{WILEY-VCH}

modes, ${ }^{[2-5]}$ with the detailed form of the structuring determining the spectral response. The use of plasmon polariton resonances in the realization of coloured pixels has been demonstrated previously via the use of several geometries of nanostructure, ${ }^{[6]}$ including periodic patterns of nanoantennas, ${ }^{[7-8]}$ metal-insulator-metal (MIM) resonators that may incorporate a continuous or perforated back plane, ${ }^{[9-14,15]}$ metallic nanocavities ${ }^{[16]}$ and arrays of sub-wavelength perforations in metal sheets. ${ }^{[17-19]}$ Diffractive coupling of light to surface plasmon polaritons ${ }^{[2,3]}$ (SPPs) via a periodically textured surface (a diffraction grating) has been demonstrated as a mechanism for optical polarization conversion. ${ }^{[20-22]}$ The arrangement of surface-relief diffraction gratings into arrays of pixels is already established as a means of creating images in optically variable devices (OVDs), with diffraction as the principal colourgenerating mechanism rather than plasmonic interaction. ${ }^{[23]}$ Such devices are now familiar as overt optical security features on banknotes and on retail products. A further goal for document security and anti-counterfeiting is the inclusion of covert images, text or patterns, ${ }^{[1]}$ whereby latent features that are invisible to the naked eye may be revealed and verified under certain specific viewing conditions. This application has been the subject of several approaches, including luminescent inks, ${ }^{[24]}$ plasmonic surface-enhanced Raman spectroscopy, ${ }^{[25]}$ controlled wetting of nanopillar arrays, ${ }^{[26]}$ polarization conversion using polymerised liquid crystals, ${ }^{[27]}$ circularly-polarized light reflectance, ${ }^{[28]}$ infrared images, ${ }^{[29]}$ magnetic field-responsive colloids ${ }^{[30]}$ and grey-scale images that are revealed by second harmonic generation. ${ }^{[31]}$ In this work we present a device that utilises plasmonic colour generation and polarization control for the realisation of optical security features. Covert fullcolour images are demonstrated in which the concealed spectral signature exists in light that has undergone an orthogonal conversion of its polarization state upon reflection from a plasmonic surface (Figure 1). A polarization-sensitive optical system is required to view the image and its colour signature, which are not visible to the naked eye at normal incidence. Nanostructured grating coupling is used to produce red, green and blue (RGB) primary colours through tailored surface plasmon interactions. The RGB colour space encloses a wide gamut of chromaticities enabling accurate colour definition using additive colour mixing. We have created a large-area $(14.4 \times 9.6 \mathrm{~mm})$ colour reproduction of the famous Mona Lisa portrait using an array of juxtaposed plasmonic pixels. Electron-beam lithography is used for the fabrication of the nanostructured surface.

Plasmonic colour generation offers potential advantages over pigment-based printing including simplified production and the use of fewer materials. Specific plasmonic techniques vary in terms of which additional desirable features they possess, as reviewed by Kristensen et 


\section{WILEY-VCH}

$a l{ }^{[6]}$ Targets for overt printed pixel methods include tunability across the visible spectrum, ${ }^{[10,32]}$ vibrant colours, ${ }^{[7,15]}$ durability, ${ }^{[33]}$ high resolution pixels ${ }^{[11,12,18]}$ and viewing angle insensitivity. ${ }^{[16,34]}$ Depending on the intended application and mode of operation, polarization independence may be considered a performance indicator, ${ }^{[32,19]}$ while alternatively a polarization-dependent response may add functionality. ${ }^{[8,13,35,36,37,38]} \mathrm{We}$ present a device architecture that extends the functionality of a polarization-dependent plasmonic response to the encoding and revelation of a full-colour invisible image. The objective is to harness beneficial features of plasmonic colour generation in the design of covert security features. The grating-based structure offers chromatic agility, scalability and the prospect of compatibility with manufacture using replication techniques. ${ }^{[39,40,41,42]}$ The use of aluminium is favoured owing to its broad plasmonic spectral range, stability, low cost and abundance. ${ }^{[43,7,9,10,32,33]}$ As a plasmonic material, aluminium has gained interest recently due to these properties. Angle insensitivity is not a goal for our device concept, as it is intended to be viewed from a fixed normal direction through an optical system.

The polarization conversion of light reflected from a surface-relief diffraction grating (see inset of Figure 1) as a result of SPP excitation occurs when the incident electric field vector has in-plane components both parallel and perpendicular to the grating grooves. ${ }^{[20-22]}$ For coupling to an SPP mode to occur a component of the electric field normal to the local surface must exist. Thus the component that has an in-plane electric field parallel to the grating grooves will be reflected without having excited an SPP, but the component with an in-plane electric field perpendicular to the grating grooves can excite an SPP, and can be subsequently re-radiated. The resonant nature of SPP excitation results in a phase shift in this re-radiated light, and the phase difference between the two components results in a change in the polarization state of the reflected light to include a constituent that is orthogonal to that of the incident polarization. Since our device is designed to work under normal incidence illumination and viewing, we adopt the notation $\mathrm{R}_{\mathrm{VH}}$ to signify the component of reflectance that is subject to conversion from vertical to horizontal linear polarization with respect to the orientation of the image. The grating vector for each pixel is orientated at $45^{\circ}$ to the vertical axis of the image.

In order to tailor the $\mathrm{R}_{\mathrm{VH}}$ spectral response of aluminium grating designs, we undertook electromagnetic simulations using the finite element method (see Experimental Section). Results of these simulations and details of how the grating pitch (period), depth and mark-pitch ratio influence the plasmonic behaviour are provided in the Supplementary Information. The simulated spectra of candidate grating profiles were converted to CIE $x y Y$ 


\section{WILEY-VCH}

coordinates, ${ }^{[44]}$ from which a set of designs providing red, green and blue $\mathrm{R}_{\mathrm{VH}}$ primary colours was identified. The grating dimensions and corresponding CIE parameters are summarised in Table 1.

The Mona Lisa painting by Leonardo da Vinci (Figure 2a) was selected for implementation in a covert image demonstrator. The $\mathrm{R}_{\mathrm{VH}}$ experimental result is shown in Figure $2 \mathrm{~b}$. The plasmonic pixels forming the image measured $24 \mu \mathrm{m}$ square and were defined comprising three juxtaposed rectangular subpixels, each providing a different primary colour and having the corresponding grating design. The subpixel RGB data of the JPEG image file were converted from the sRGB colour space ${ }^{[45]}$ to $\mathrm{R}_{\mathrm{VH}} \mathrm{RGB}$ values using a standard $\operatorname{algorithm}^{[46]}$. These values were applied to the pixel array in which the relative intensity of each primary colour within a specific pixel was controlled by adjusting the area of the grating. Unused space contained flat metal, which does not convert the polarization and therefore does not contribute to the reflected spectrum. This method of pixel definition allows the independent selection of any chromaticity and relative intensity within the $\mathrm{R}_{\mathrm{VH}}$ colour space using additive colour mixing, and as such the technique is not limited to a restricted palette of discrete colours. A layout file in GDS II format was used to specify the electron-beam write pattern in the fabrication process (see Experimental Section). Dielectric encapsulation of the nanostructured metal surface was provided by a glass superstrate. The maximum relative areas of the red, green and blue subpixels are given respectively by the factors $N_{R}=1.1065$, $N_{G}=0.8817$ and $N_{B}=1.0118$. This combination yields white corresponding to the standard illuminant E. The image resolution was 402 by 599 pixels, giving overall image dimensions of $14.4 \times 9.6 \mathrm{~mm}$. The layout additionally included $1.5 \mathrm{~mm}$-square patches of each of the RGB grating designs and a white patch for spectral measurement. The experimentallyobtained $\mathrm{R}_{\mathrm{VH}}$ reproduction of the Mona Lisa portrait (Figure 2b) shows the covert image as revealed by the polarization-sensitive optical system (see Figure 4, Experimental Section), in which the device is illuminated with vertically-polarized white light and the surface is imaged through a second polarizer arranged to transmit horizontally-polarized light. Supplementary video 1 shows the transfer of this device between unpolarized illumination and the polarization-sensitive optical system. The image exhibits realistic colour reproduction, while the pixel resolution is sufficient to show detail of the artwork. The entire sample, containing the Mona Lisa portrait and the four coloured patches is shown under unpolarized illumination in Figures $2 \mathrm{c}$ to $2 \mathrm{e}$. A general view of the sample in daylight for a near-normal viewing angle (Figure 2c) demonstrates that the image is not overt under these viewing conditions. Some contrast is apparent between the reflectance of grating regions and the flat metal regions, 


\section{WILEY-VCH}

which is due to a combination of the optical absorption of the SPPs and some coupling to the \pm 1 diffraction orders that propagate within the dielectric overlayer for some wavelengths. It may be envisioned that the presence of the image could be further concealed by reducing this contrast by other means, some of which are now being explored. Figures $2 \mathrm{~d}$ and $2 \mathrm{e}$ show the sample photographed under directional unpolarized illumination, orientated approximately $60^{\circ}$ from normal and with azimuth aligned to the grating vector at $\phi=0^{\circ}$. When the viewing angle is approximately co-aligned with the illumination (Figure 2d), the image and colour patches become visible in the -1 diffraction order. For other viewing angles, such as $\phi=-90^{\circ}$ (Figure 2e), the diffracted light is not visible. The -1 diffraction order is the only free-space propagating order, and it can only be viewed with representative colours over a very limited range of illumination and viewing angles. In the context of optical security features, this property adds optically variable device functionality. A perfect match to the design-specified colours, as shown in Figure $2 \mathrm{~b}$, can only be seen in the $\mathrm{R}_{\mathrm{VH}}$ image, affording a 'forensiclevel' security feature. The arrangement of area-weighted RGB sub-pixels in $24 \mu \mathrm{m}$-square pixels is shown in the high-magnification $R_{V H}$ image of Figure $2 f$. Further detail of the nanostructured surface is shown in a scanning electron micrograph of a bare-metal test sample (Figure $2 \mathrm{~g}$ ). The diffraction gratings are imaged in plan view at the conjunction of red (left) and green (right) subpixels. The grating ridges appear brighter than the grooves.

Figure 3 shows simulations (Figure 3a) and experimental results (Figure 3b) (see Experimental Section) for the $\mathrm{R}_{\mathrm{VH}}$ spectra of the RGB and white colour designs and patches. The CIE $x y$ coordinates of these spectra are plotted on the chromaticity chart in Figure $3 \mathrm{c}$. The white patch provides an example of additive colour mixing using the same RGB primary colours that are implemented in the Mona Lisa image. A good agreement between the simulations and measurements in the shapes and positions of the corresponding spectra is seen, while the experimental results show weaker signals than those expected by the models. This discrepancy may be attributable to tolerances in the fabricated grating dimensions, particularly the grating depth, and the influence of composition and granularity upon the permittivity of the metal. ${ }^{[43]}$ A photograph of the $R_{V H}$ coloured patches is shown in Figure $3 \mathrm{~d}$. Note that in each of the RGB patches the entire area is filled with grating, whereas in an equivalent image pixel of those colours a maximum of approximately one third of the pixel area would be filled. Consequently, the white patch appears grey for the camera exposure used as its signal is one third of the corresponding RGB sum. Flat metal surrounding the patches appears black as it does not convert the polarization. This result confirms the generation of bright, vivid colours and high contrast using this polarization conversion 


\section{WILEY-VCH}

mechanism. The selected chromaticities strike a balance between the conflicting goals of enclosing a very broad gamut of chromaticities and achieving bright pixels. This latter aim is not served by the narrow spectra that are associated with highly-saturated colours.

This work has demonstrated the preparation of covert images with accurate, vivid, full-colour reproduction. The work has combined plasmonic colour production, polarization manipulation and pixel definition to achieve its objective. An electron beam lithography process that was developed to fabricate the nano-structured metal film demonstrates a route to the origination of devices, while the prospect of compatibility with manufacture using existing replication technologies is suggested. This plasmonic technique promises utility in anticounterfeiting applications, for example by deployment on high-value documents or product packaging. The method demonstrated here offers rapid authentication using an optical instrument, while the chromatic fidelity demonstrated provides the potential for adaptable, complex covert signatures to serve future needs for optical security features.

\section{Experimental Section}

Fabrication: The layout was written into a $45 \mathrm{~nm}$-thick layer of $100 \mathrm{~K}$ A4 polymethylmethacrylate (PMMA) resist on a glass substrate using a Nanobeam NB4 electron beam system. An e-beam dose of $4.5 \mathrm{Cm}^{-2}$ was applied with a beam current of $4 \mathrm{nA}$. A 10 $\mathrm{nm}$-thick layer of aluminium was deposited by thermal evaporation prior to e-beam exposure to prevent substrate charging, and was removed prior to development using tetramethyl ammonium hydroxide. The exposed resist was developed in a 15:5:1 solution of IPA:MIBK:MEK for $45 \mathrm{~s}$, rinsed for $60 \mathrm{~s}$ in IPA, and blow-dried in $\mathrm{N}_{2}$. The surface profile of the patterned PMMA was then transferred to metal and inverted by coating the patterned surface with a $110 \mathrm{~nm}$-thick layer of aluminium by thermal evaporation. In operation, the image is viewed through the glass substrate, which therefore functions as a superstrate. Consequently, the plasmonic aluminium surface is not exposed to air.

Image recording: $\mathrm{R}_{\mathrm{VH}}$ images of the Mona Lisa sample (Figure $2 \mathrm{~b}$ ) and the primary-coloured test patches (Figure 3d) were recorded using the setup shown in Figure 4. Light from a white LED source was vertically polarized by a Polaroid sheet and directed to the $\mathrm{R}_{\mathrm{VH}}$ device by a 50:50 beamsplitter. Reflected light was transmitted through the beamsplitter and the horizontally polarized component was transmitted by a second polarizer. A Nikon D3200 digital single lens reflex (DSLR) camera, fitted with a Nikkor 18-55 mm lens and a $36 \mathrm{~mm}$ macro extension tube was used. The white balance of the source and optical system was 


\section{WILEY-VCH}

calibrated to the D65 standard illuminant by measurement of the co-polarized reflectance signal with the sample replaced by an aluminium mirror. For the images of the sample viewed under unpolarized illumination (Figure 2c, $2 \mathrm{~d}$ and 2e), the white balance was calibrated using a sheet of white paper. An $R_{V H}$ image of the subpixel arrangement (Figure 2f) was recorded using the polarising microscope described below, fitted with a $\times 50$ objective. For the illumination conditions in this high-magnification configuration, the observed colours should be considered for indication only.

Spectral measurements: The $\mathrm{R}_{\mathrm{VH}}$ spectra of the fabricated test patches were measured using a Zeiss Axioskop2 polarising microscope fitted with a low-magnification objective, with the illumination and viewing paths containing linear polarizers with azimuth angles set orthogonally to each other, and the grating vector of the device orientated at the intermediate $45^{\circ}$ angle. The microscope was fitted with a Zeiss XBO75 broadband optical source and an Ocean Optics USB2000 fibre-coupled optical spectrometer. A calibration was performed to take account of the source spectrum and transmission through the optical system.

Spectral simulations: The polarization-dependent reflectance was modelled using the proprietary software package Ansys HFSS, which implements the Finite Element Method. The wavelength-dependent complex permittivity data for aluminium reported by Rakić ${ }^{[47]}$ were implemented in the model, and a lossless refractive index of 1.5 was assigned to the dielectric overlayer.

\section{Supporting Information}

Supporting Information is available from the Wiley Online Library or from the author.

\section{Acknowledgements}

This work was supported by the UK Engineering and Physical Sciences Research Council (EPSRC) Knowledge Transfer Account programme grant EP/H50012X/1, and by QinetiQ Ltd. The authors would like to thank Dr Andrew Treen for useful discussions.

\section{References}

[1] L. Li, Business Horizons 2013, 56, 167.

[2] W. L. Barnes, A. Dereux, T. Ebbesen, Nature 2003, 424, 824.

[3] H. Raether, Surface Plasmons on Smooth and Rough Surfaces and on Gratings, Springer Verlag, Berlin 1988.

[4] S. Lal, S. Link, N. J. Halas, Nature Photon. 2007, 1, 641.

[5] N. Meinzer, W. L. Barnes, I. R. Hooper, Nature Photon. 2014, 8, 889. 


\section{WILEY-VCH}

[6] A. Kristensen, J. K. W. Wang, S. I. Bozhevolnyi, S. Link, P. Norlander, N. J. Halas, N. Asger Mortensen, Nat. Rev. Mat. 2016, 2, 16088.

[7] J. Olson, A. Manjavacas, L. Liub, Wei-Shun Chang, B. Foerster, N. S. King, M. W. Knight, P. Nordlander, N. J. Halas, S. Link, PNAS 2014, 111, 14348.

[8] T. Ellenbogen, K. Seo, K. B. Crozier, Nano Lett. 2012, 12, 1026.

[9] T. D. James, P. Mulvaney, A. Roberts, Nano Lett. 2016, 16, 3817.

[10] S. J. Tan, L. Zhang, D. Zhu, X. M. Goh, Y. M. Wang, K. Kumar, C.-W. Qiu, J. K. W. Yang, Nano Lett. 2014, 14, 4023.

[11] A. S. Roberts, A. Pors, O. Albrektsen, S. I. Bozhevolnyi, Nano Lett. 2014, 14, 783.

[12] K. Kumar, Huigao Duan, R. S. Hegde, S. C. W. Koh, J. N. Wei, J. K. W. Yang, Nature Nanotech. 2012, 7, 557.

[13] X. M. Goh, Y. Zheng, S. J. Tan, K. Kumar, C.-W. Qiu, J. K. W. Yang, Nature Commun. 2014, 5, 5361.

[14] T. Xu, Y. K. Wu, X. G. Luo, L. J. Guo, Nature Commun. 2010, 1, 59.

[15] H. Wang, X. Wang, C. Yan, H. Zhao, J. Zhang, C. Santschi, O. J. F. Martin, ACS. Nano. 2017, 11, 4419.

[16] Y.-K. R. Wu, A. E. Hollowell, C. Zhang, L. J. Guo, Sci. Rep. 2013, 3, 1194.

[17] S. Yokogawa, S. P. Burgos, H. A. Atwater, Nano Lett. 2012, 12, 4349.

[18] R. Rajasekharan, E. Balaur, A. Minovich, S. Collins, T. D. James, A. Djalalian-Assl, K. Ganesan, S. Tomljenovic-Hanic, S. Kandasamy, E. Skafidas, D. N. Neshev, P. Mulvaney, A. Roberts and S. Prawer, Sci. Rep. 2014, 4, 6435.

[19] D. Inoue, A. Miura, T. Nomura, H. Fujikawa, K. Sato, N. Ikeda, D. Tsuya, Y. Sugimoto, Y. Koide, Appl. Phys. Lett. 2011, 98, 093113.

[20] G. P. Bryan-Brown, J. R. Sambles, M. C. Hutley, J. Mod. Opt. 1990, 37, 1227.

[21] R. A. Watts, J. R. Sambles, Opt. Comm. 1997, 140, 179.

[22] I. R. Hooper, J. R. Sambles, Opt. Lett. 2002, 27, 2152.

[23] R. A. Lee, P. W. Leech, Microelectron. Eng. 2000, 53, 513.

[24] T. Blumenthal, J. Meruga, P. S. May, J. Kellar, W. Cross, K. Ankireddy, S. Vunnam, Q. N. Luu, Nanotech. 2012, 23, 185305.

[25] Y. Cui Y, R. S. Hegde, I. Y. Phang, H. K. Lee, X. Y. Ling, Nanoscale 2014, 6, 282.

[26] K. G. Lee, B. G. Choi, B. I. Kim, T. Shy, M. S. Oh, S. G. Im, S.-J. Chang, T. J. Lee, N. A. Kotov, S. J. Lee, Adv. Mater. 2014, 26, 6119.

[27] S. Suzuki, H. Kaneiwa, I., United States Patent Application US2010/0119738, 2010.

[28] Y. P. Zhang, V. P. Chodavarapu, A. G. Kirk, M. P. Andrews, J. Nanophoton. 2012, 6, 063516.

[29] M. Makhsiyan, P. Bouchon, J. Jaeck, J.-L. Pelouard, R. Haïdar, Appl. Phys. Lett. 2015, 107, 251103.

[30] H. Hu, J. Tang, H. Zhong, Z. Xi, C. Chen, Q. Chen, Sci. Rep. 2013, 3, 1484.

[31] F. Walter, G. Li, C. Meier, S. Zhang, T. Zentgraf, Nano. Lett. 2017, 17, 3171.

[32] R. Mudachathi, T. Tanaka, Sci. Rep. 2017, 7, 1199.

[33] J. S. Clausen, E. Høilund-Nielsen, A. B. Christiansen, S. Yazdi, M. Grajower, H. Taha, U. Levy, A. Kristensen, N. A. Mortensen, Nano. Lett. 2014, 14, 4499.

[34] K. Mao, W. Shen, C. Yang, X. Fang, W. Yuan, Y. Zhang, X. Liu, Sci. Rep. 2016, 6, 19289. 


\section{WILEY-VCH}

[35] L. Duempelmann, A. Luu-Dinh, B. Gallinet, L. Novotny, ACS Photon. 2015, 3, 190.

[36] Y.-W. Huang, W. T. Chen, W.-Y. Tsai, P. C. Wu, C.-M. Wang, G. Sun, D. P. Tsai, Nano Lett. 2015, 15, 3122.

[37] Z. Li, A. W. Clark, J. M. Cooper, ACS. Nano. 2016, 10, 492.

[38] I. Koirala, V. R. Srestha, C.-S. Park, S.-S. Lee, D.-Y. Choi, Sci. Rep. 2017, 7, 40073.

[39] P. W. Leech, R. A. Lee, J. Mater. Sci. 2007, 42, 4428.

[40] F. Lütolf, M. Stalder, O. J. F. Martin, Adv. Opt. Mat. 2015, 3, 1793-1799.

[41] S. H. Ahn, L. J. Guo, Adv. Mat. 2008, 20, 2044-2049.

[42] N. Kooy, K. Mohamed, L. T. Pin, O. S. Guan, Nanoscale Res. Lett. 2014, 9, 320.

[43] M. W. Knight, N. S. King, L. Liu, H. O. Everitt, P. Nordlander, N. J. Halas, ACS Nano 2014, 8, 834 .

[44] Commission Internationale de l'Eclairage proceedings, 1931. Cambridge University Press, 1932.

[45] IEC 61966-2-1, Geneva, Switzerland, 1999.

[46] Society of Motion Picture and Television Engineers, Derivation of Basic Television Color Equations, SMPTE Recommended Practice RP 177-1993, 1993.

[47] A. D. Rakić, Appl. Opt. 1995, 34, 4755.

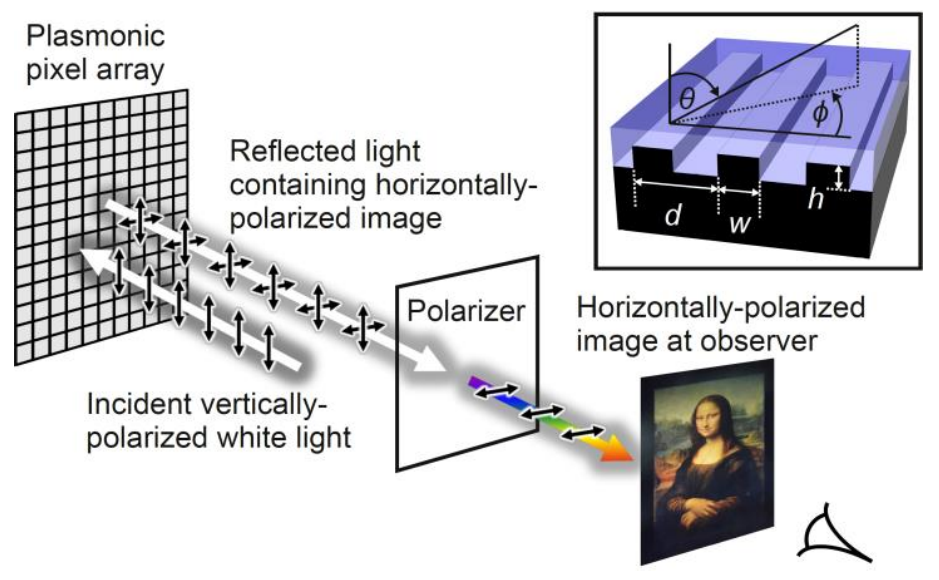

Figure 1. Concealed image generation using polarization conversion at a plasmonic pixel array. Schematic outlining the principles of image concealment and revelation. Inset: schematic of a portion of a metallic grating with a dielectric overlayer, indicating the polar angle $\theta$, the azimuth angle $\phi$ with respect to the grating vector, the grating depth $h$, the pitch $d$ and the ridge width $w$. The mark-pitch ratio $r$ is given by $w / d$. The grating vector is orientated at $45^{\circ}$ to the vertical axis of the image. 


\section{WILEY-VCH}

Mona Lisa photograph

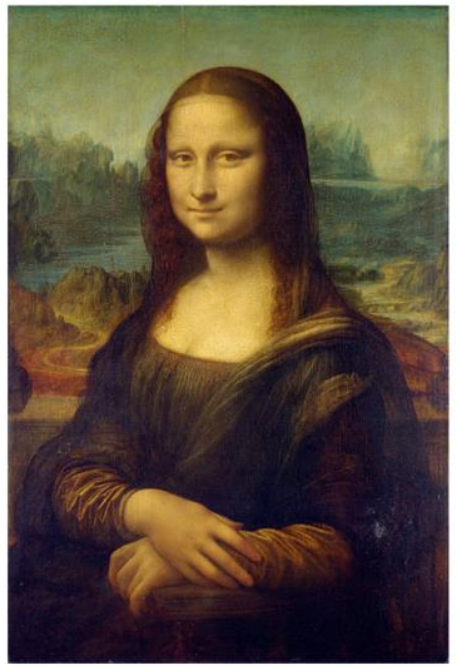

Experimental result

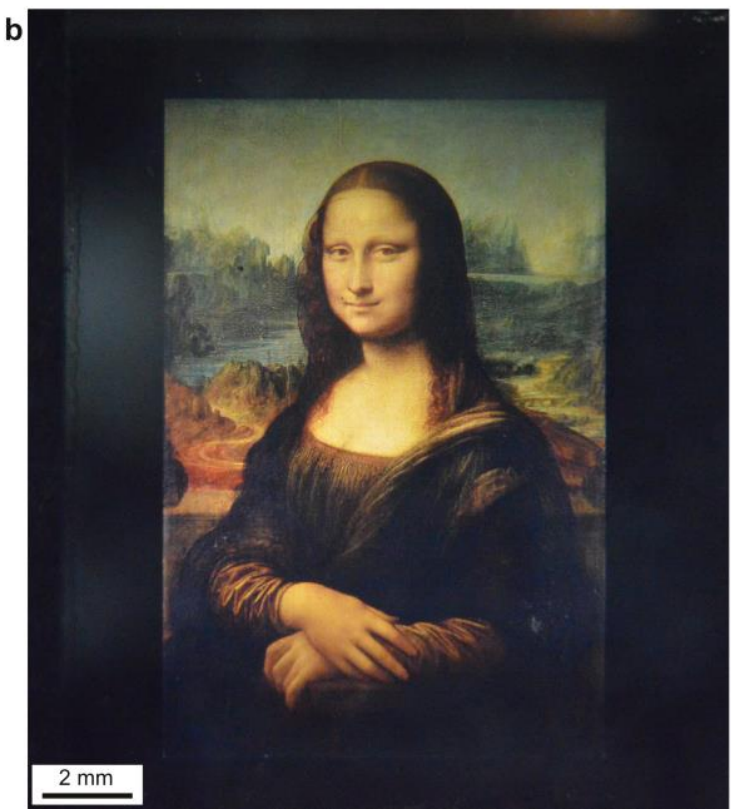

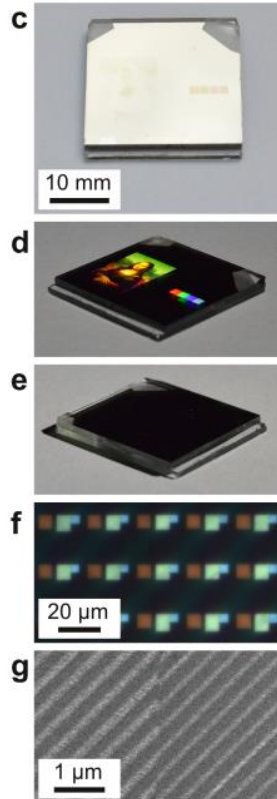

Figure 2. Experimental results showing images of an $R_{v H}$ device under various illumination conditions.

a, A photograph of the Mona Lisa portrait (La Joconde), containing the colour data that were used in the design of the covert image device. Reproduced with permission (C) C2RMF / Elsa Lambert. b, Experimental result showing an $\mathrm{R}_{\mathrm{VH}}$ image of the Mona Lisa portrait. The hidden image was revealed using an optical system providing vertically-polarized illumination at normal incidence and viewing through a linear polarizer arranged to transmit only horizontally-polarized light. The portrait measures $14.4 \times 9.6 \mathrm{~mm}$. c, A general view of the device in daylight for a near-normal viewing angle. The image is not visible under these conditions. Some contrast is apparent between the reflectance of grating regions and the flat metal regions, which is due to the optical loss in the patterned regions being greater than that from flat metal. d,e, Two views of the device under directional unpolarized illumination. A white LED source was orientated at an elevation angle $\theta$ of approximately $60^{\circ}$ from normal, with an azimuth $\phi$ of zero, i.e. aligned to the grating vector. d, Viewing angle approximately co-aligned with the illumination. The Mona Lisa portrait and the red, green, blue and white test patches are visible in the -1 diffraction order. e, Viewing angle of approximately $\theta=60^{\circ}$ in elevation and $\phi=-90^{\circ}$ in azimuth, for which diffracted light is not visible. f, A highmagnification $R_{V H}$ image showing the arrangement of area-weighted RGB sub-pixels in 24 $\mu \mathrm{m}$-square pixels. The colours shown in figure $2 \mathrm{f}$ should be considered for indication only due to the illumination conditions associated with the high-magnification configuration. $\mathbf{g}$, Scanning electron micrograph of a bare-metal test sample, showing the diffraction gratings in plan view at the conjunction of red (left) and green (right) subpixels. The grating ridges appear brighter than the grooves. 


\section{WILEY-VCH}
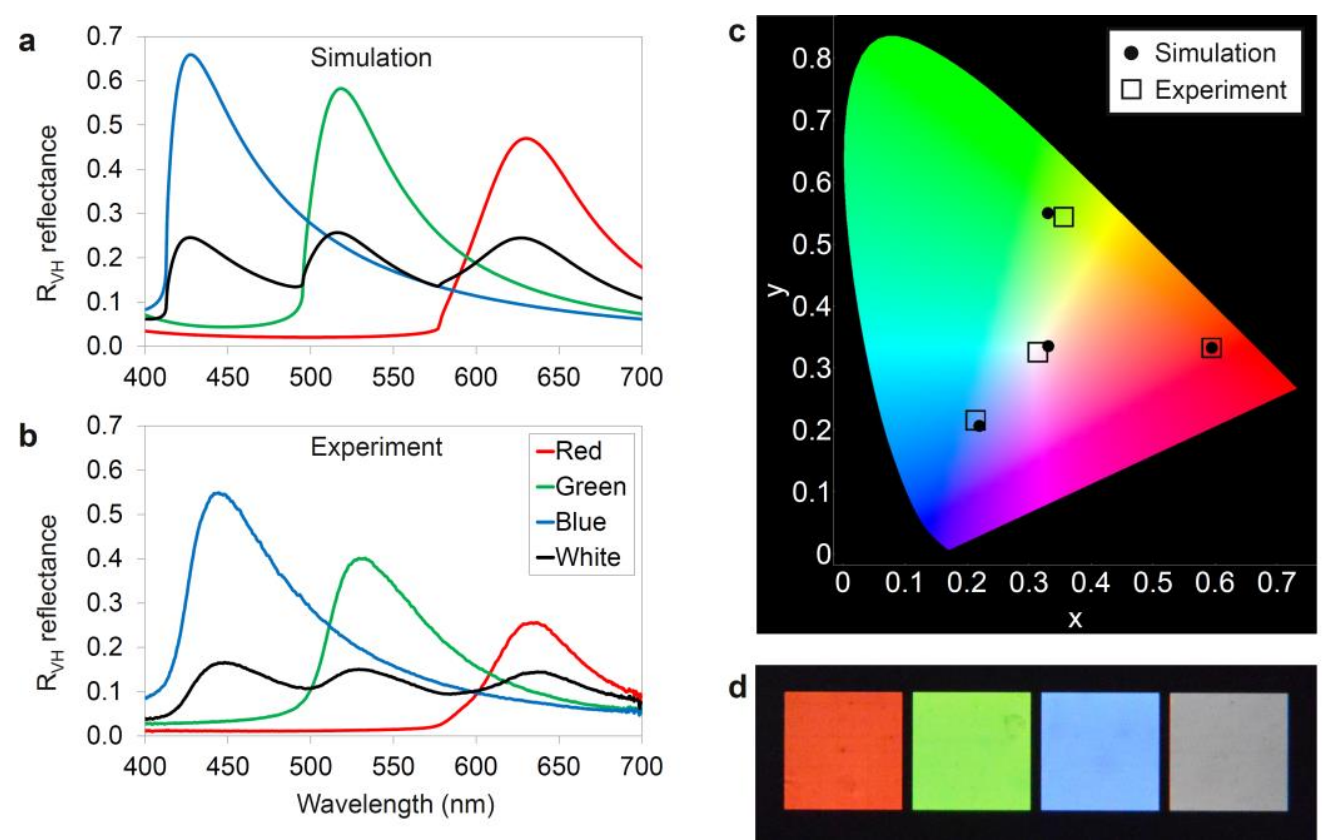

Figure 3. RvH reflectance spectra and chromaticities for the RGB primary colours; simulations and experimental results. a, Simulations of the $\mathrm{R}_{V H}$ reflectance spectra at normal incidence for red, green and blue subpixel grating designs and their weighted combination in a white pixel. b, Experimental results showing the $\mathrm{R}_{\mathrm{VH}}$ reflectance spectra for red, green, blue and white $\mathrm{R}_{\mathrm{VH}}$ colour patches at normal incidence. c, The chromaticities of the RvH primary colours and white pixel plotted on the CIE 1931 chart; simulations (circles) and experimental results (squares). d, An experimental result showing a calibrated image of the $\mathrm{R}_{\mathrm{VH}}$ colour patches viewed through a V-H polarizing optical system (see Figure 4). Each patch measures $1.5 \mathrm{~mm}$ square. The white patch appears grey for the camera exposure used because each of the RGB patches is filled entirely with grating, whereas in an equivalent image pixel of those colours a maximum of approximately one third of the pixel area would be filled. Accordingly, the white patch is one third as bright as the corresponding RGB sum.

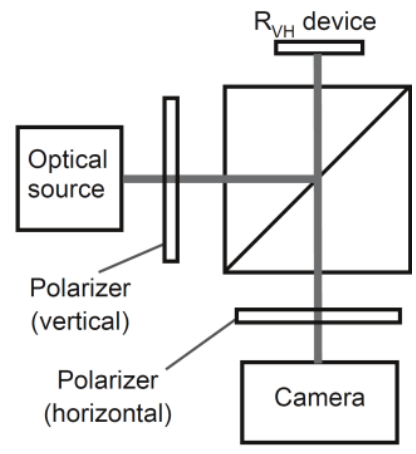

Figure 4. Schematic of the experimental setup for $R_{v H}$ image recording. 


\section{WILEY-VCH}

Table 1. Grating dimensions and chromaticity data for the selected RvH RGB primary colours. A rectangular grating profile with a depth of $45 \mathrm{~nm}$ is specified for each design. Aluminium is specified for the metal and a dielectric encapsulation of refractive index 1.5 is applied.

\begin{tabular}{lccccc}
\hline $\begin{array}{l}\text { Primary } \\
\text { colour }\end{array}$ & $\begin{array}{c}\text { Grating } \\
\text { pitch [nm] }\end{array}$ & $\begin{array}{c}\text { Mark-pitch } \\
\text { ratio }\end{array}$ & $x$ & $y$ & $Y$ \\
\hline Red & 385 & 0.475 & 0.597 & 0.331 & 0.107 \\
Green & 330 & 0.35 & 0.333 & 0.548 & 0.320 \\
Blue & 275 & 0.3 & 0.222 & 0.205 & 0.172 \\
\hline
\end{tabular}

